\title{
AVALIAÇÃO DO USO DE PSICOFÁRMACOS DURANTE O PERÍODO DE GRAVIDEZ E LACTAÇÃO
}

\author{
EVALUATION OF THE USE OF PSYCHOACTIVE DRUGS DURING THE PERIOD OF PREGNANCY AND LACTATION
}

\author{
AMORIM, Isadora¹. RODRIGUES, Luanaํ. ROCHA. Marcela³, BARROS, Mário \\ ${ }^{1}$ FAHESP - Faculdade de Ciências Humanas, Exatas e da Saúde do Piauí. IESVAP - Instituto de Educação Superior do Vale do Parnaíba \\ S.A. FAHESP/IESVAP, Parnaíba, Brasil
}

\section{RESUMO}

Os psicofármacos se destacam por constituírem uma classe muito utilizada pelas mulheres no período gravídico-puerperal, já que estas estão mais vulneráveis a alterações físicas, hormonais, psíquicas e de inserção social. O contato do feto com essas drogas pode provocar consequências como teratogenicidade, 0 que leva ao risco de malformações congênitas; toxicidade neonatal, devido a exposição direta do feto com o fármaco; e sequelas neurológicas, comportamentais, psicológicas e cognitivas à longo termo. Assim, cabe ao profissional de saúde avaliar os riscos e os benefícios do uso de determinadas drogas durante esses períodos, buscando uma melhor escolha. O presente estudo tem como objetivo realizar uma revisão integrativa acerca do uso de psicofármacos no decorrer da gestação e lactação e analisar as evidências cientificas sobre seus efeitos na gestação e lactação. Este trabalho trata-se de uma pesquisa bibliográfica qualitativa, cujo objetivo é exploratório. Foram averiguadas informações e elucidadas questões sobre o tema, visando uma abordagem analítica do conteúdo em busca de contradições e unanimidade entre os autores, através de um levantamento das publicações. Foi realizada uma síntese dos resultados de pesquisas produzidas com o intuito de avaliar do uso de psicofármacos no decorrer da gestação e lactação e a partir de análise definiu-se os melhores a serem utilizados. A busca bibliográfica foi baseada em consultas por via eletrônica ao material de bibliotecas virtuais e bancos de dados como BVS, SCIELO, LILACS e Google acadêmico nos idiomas português e inglês, abrangendo artigos publicados no período compreendido entre 2005 e 2018 existente. A partir da análise e exploração, foram selecionados 16 artigos, os quais constituíram o corpus do estudo. Na literatura, a segurança do uso dos psicofármacos durante a gestação e lactação ainda não foi estabelecida. Entretanto, alguns estudos apontaram alguns fármacos menos teratogênicos, tanto para o bebê como para a mãe.

Palavras-chave: Gravidez. Lactação. Psicofármacos.

\begin{abstract}
Psychopharmaceuticals stand out because they are a class widely used by women in the pregnancy-puerperal period, since they are more vulnerable to physical, hormonal, psychic and social insertion changes. The contact of the fetus with these drugs can have consequences such as teratogenicity, which leads to the risk of congenital malformations; neonatal toxicity due to direct exposure of the fetus to the drug; and long-term neurological, behavioral, psychological and cognitive sequelae. Thus, it is up to the health professional to assess the risks and benefits of using certain drugs during these periods, seeking a better choice. To carry out a bibliographic review about the use of psychoactive drugs during pregnancy and lactation and from the analysis to define the best to be used. This work is a qualitative bibliographical research whose objective is exploratory. Information was analyzed and elucidated questions on the topic addressed, aiming at an analytical approach of the content in search of contradictions and unanimity among the authors, through a survey of the publications. A synthesis of the results of researches produced with the intention of evaluating the use of psychoactive drugs during gestation and lactation was performed and from the analysis the best ones to be used were defined. The bibliographic search was based on electronic queries to the material of virtual libraries and databases such as BVS, SCIELO, LILACS and Google academic in Portuguese and English, covering articles published in the period between 2005 and 2018. From the analysis and exploration, 16 articles were selected, which constituted the corpus of the study. In the literature, the safety of psychoactive drugs during gestation and lactation has not been established. However, some studies have pointed to some less teratogenic drugs for both the baby and the mother.
\end{abstract}

Keywords: Pregnancy. Lactation. Psychopharmaceuticals.

Correspondência: AMORIM, Isadora e-mail: isadorinhaamorim35@hotmail.com

Recebido: 31 maio 2019

Aprovado: 12 jul. 2019 


\section{INTRODUÇÃO}

Uma atenção pré-natal e puerperal de qualidade e humanizada é fundamental para a saúde materna e neonatal. Esta se dá por meio da incorporação de condutas acolhedoras e sem intervenções desnecessárias; do fácil acesso a serviços de saúde de qualidade, com ações que integrem todos os níveis da atenção: promoção, prevenção e assistência à saúde da gestante e do recém-nascido, desde 0 atendimento ambulatorial básico ao atendimento hospitalar para alto risco ${ }^{1}$.

Para que este período transcorra com segurança, são necessários cuidados da própria gestante, do parceiro, da família e, especialmente, dos profissionais de saúde 2 . No período gravídico ocorrem várias mudanças físicas, hormonais e psicológicas e por esse motivo podem advir transtornos psíquicos, ocasionando danos não só à saúde materna, mas pode comprometer também a qualidade da relação mãe-criança e o desenvolvimento do bebê3.

$\mathrm{Na}$ fase puerperal, a mulher encontra-se exposta a maiores riscos de aparecimentos de transtornos mentais em relação a outras fases da vida, uma vez que as defesas físicas e psicossociais são direcionadas a proteção e vulnerabilidade do bebêt ${ }^{4}$ O período gravídicopuerperal é uma situação do ciclo de vida das mulheres que demanda uma maior atenção, por ser um momento em que frequentemente encontramos importantes alterações psicofisiológicas e psicopatológicas. Alterações e oscilações do humor são frequentes nesses períodos ${ }^{5}$.

A atenção à saúde mental das mulheres ainda é deficiente, visto que atuam somente em serviços específicos e não possuem uma visão holística da paciente, pelo fato de não abrangerem questões como gestação e lactação. A gravidez não protege as pacientes contra a ocorrência, a recorrência ou a exacerbação de transtornos psiquiátricos. Ao contrário disso, esse período é considerado o de maior prevalência de transtornos mentais na mulher, principalmente no primeiro e no terceiro trimestre de gestação e nos primeiros 30 dias de puerpério ${ }^{6}$.

A atuação multiprofissional, nesse contexto, destacase ao passo que contribui para um diagnóstico precoce e consequente avaliação de risco-benefício, influenciando na escolha do fármaco mais adequado para o período em que a mulher se encontra. No entanto, o atendimento é pouco abrangente e pouco eficaz às mulheres com transtorno mental, visto que os profissionais da atenção básica são pouco instruídos acerca de informações e ações que devem estar ao alcance desse público ${ }^{7}$.

\section{MÉTODOS}

Este trabalho trata-se de uma pesquisa bibliográfica qualitativa, cujo objetivo é exploratório. Foi realizada uma síntese dos resultados de pesquisas produzidas com o intuito de avaliar do uso de psicofármacos no decorrer da gestação e lactação e a partir de análise definiu-se os melhores a serem utilizados. Foram averiguadas informações e elucidadas questões sobre o tema abordado, visando uma abordagem analítica do conteúdo em busca de contradições e unanimidade entre os autores, através de um levantamento das publicações.

A busca bibliográfica foi baseada em consultas por via eletrônica ao material de bibliotecas virtuais e bancos de dados como Biblioteca Virtual de Saúde (BVS), Scientific Eletronic Library Online (SCIELO), Literatura Latinoamericana e do Caribe em Ciências da Saúde (LILACS) e Google acadêmico nos idiomas português e inglês, abrangendo artigos publicados no período compreendido entre 2005 e 2018 existentes. Conforme ilustra figura 1, foram inclusas 16 pesquisas.

Figura 1. Estudos selecionados

Os descritores que foram utilizados nesta revisão são "pré-natal", "gravidez", "lactação", "saúde mental" e

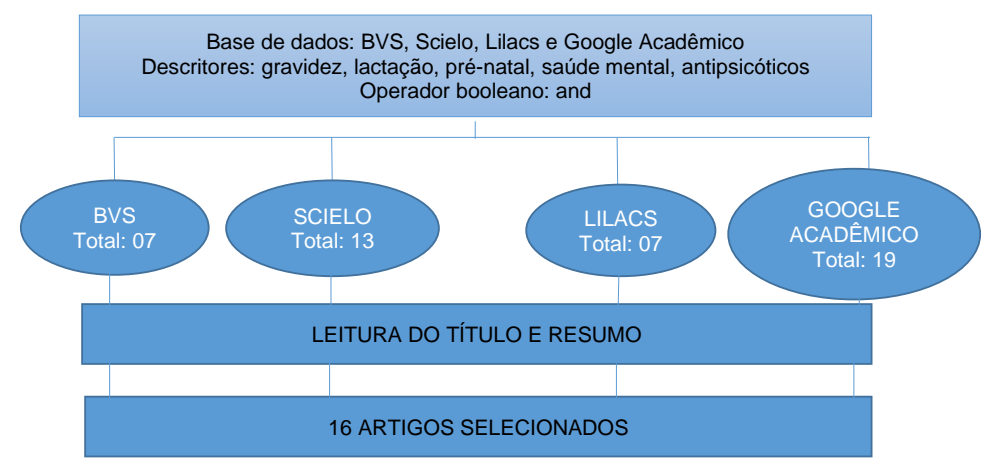

"antipsicóticos", em inglês definidos por "prenatal", "pregnancy", "lactation", "mental health" e "antipsychotics". Utilizou-se o operador booleano AND, com intuito de correlacionar esses descritores apresentados nos bancos de dados citados anteriormente para validá-los.

Como estratégia para limitação da busca adotou-se artigos originais de revisão bibliográfica e estudos de caso, assim como trabalhos os quais respondiam às questões: "A partir da análise dos psicotrópicos utilizados por gestantes e lactantes, quais os mais adequados para esses períodos?" e "Quais as reais consequências que 0 uso de psicofármacos ocasiona durante a gravidez e lactação para a mãe e o feto?". Além disso, outro critério de inclusão foi a escolha de pesquisas publicadas em dois idiomas, português e inglês. Realizou-se a leitura completa dos artigos selecionados para identificar os critérios de exclusão e, assim, diminuiu-se o risco de vieses na pesquisa. Foram excluídos os estudos realizados com mulheres que não fizeram uso de algum medicamento durante o período de gravidez e lactação, os que não abordavam a saúde mental e aqueles que não foram publicados no período determinado.

\section{RESULTADOS E DISCUSSÃO}

A Tabela 1 apresenta a síntese dos estudos incluídos na revisão, os quais constituíram o corpus do estudo e 
representaram a essência para a elaboração dos resultados, discussão, e respectiva conclusão sobre a temática do uso de psicofármacos durante o período de gravidez e lactação.

$\mathrm{Na}$ fase puerperal, a mulher encontra-se exposta a maiores riscos de aparecimentos de transtornos mentais em relação a outras fases da vida, uma vez que as defesas físicas e psicossociais são direcionadas a proteção e vulnerabilidade do bebêt.

Fernandes ${ }^{8}$ afirma que o uso de medicamentos nessas etapas da vida - gravidez e puerpério - é comum e consiste em uma problemática, principalmente relacionada à segurança e eficácia da droga. Ainda, há poucos estudos que descrevem e qualificam os riscos da utilização de muitos fármacos durante a gestação e lactação, inclusive de psicofármacos.

Tabela 1. Caracterização das publicações quanto aos autores, ano, título e periódico.

\begin{tabular}{|c|c|c|}
\hline Autor/Ano & Título & Periódico/Livro \\
\hline Alberto; Cacellier, 2017 & $\begin{array}{l}\text { Antidepressivos na } \\
\text { ginecologia e obstetrícia }\end{array}$ & $\begin{array}{l}\text { Revista Psychiatry Online } \\
\text { Brasil }\end{array}$ \\
\hline Anton; Mariano, 2017 & $\begin{array}{l}\text { Avaliação da segurança no } \\
\text { uso de antidepressivos na } \\
\text { amamentação }\end{array}$ & $\begin{array}{l}\text { Revista Interdisciplinar de } \\
\text { Estudos em Saúde }\end{array}$ \\
\hline Blaya et al., 2005 & $\begin{array}{l}\text { Diretrizes para o uso de } \\
\text { psicofármacos durante a } \\
\text { gestação e lactação }\end{array}$ & $\begin{array}{l}\text { Psicofármacos: consulta } \\
\text { rápida }\end{array}$ \\
\hline Brasil, 2014 & $\begin{array}{l}\text { Amamentação e uso de } \\
\text { medicamentos e outras } \\
\text { substâncias }\end{array}$ & $\begin{array}{l}\text { Secretaria de Atenção à } \\
\text { Saúde }\end{array}$ \\
\hline Costa et al., 2010 & $\begin{array}{l}\text { Uso de psicofármacos na } \\
\text { gravidez }\end{array}$ & Acta Obstet Ginecol Port \\
\hline Duncan et al., 2014 & $\begin{array}{l}\text { Medicamentos e outras } \\
\text { exposições na gestação e } \\
\text { na amamentação }\end{array}$ & $\begin{array}{l}\text { Medicina Ambulatorial: } \\
\text { condutas de atenção } \\
\text { primária baseadas em } \\
\text { evidências }\end{array}$ \\
\hline Ferrão et al., 2015 & $\begin{array}{l}\text { Psicofármacos na gravidez } \\
\text { e lactação }\end{array}$ & $\begin{array}{l}\text { Psicofármacos: consulta } \\
\text { rápida }\end{array}$ \\
\hline Fernandes, 2013 & $\begin{array}{l}\text { Assistência farmacêutica } \\
\text { em gestantes }\end{array}$ & $\begin{array}{l}\text { Trabalho de conclusão de } \\
\text { curso e graduação em } \\
\text { Farmácia-Bioquímica }\end{array}$ \\
\hline Gusso; Lopes, 2019 & Medicamentos e gestação & $\begin{array}{l}\text { Tratado de Medicina da } \\
\text { Família e Comunidade: } \\
\text { princípios, formação e } \\
\text { prática }\end{array}$ \\
\hline Marlon; Guedes, 2017 & $\begin{array}{l}\text { Uso de medicamentos } \\
\text { durante a lactação: um } \\
\text { fator para suspensão do } \\
\text { aleitamento materno }\end{array}$ & $\begin{array}{l}\text { Revista de Pesquisa } \\
\text { Interdisciplinar }\end{array}$ \\
\hline Moreira et al., 2016 & $\begin{array}{l}\text { Incidência de transtornos } \\
\text { psíquicos na gravidez e } \\
\text { puerpério em mulheres de } \\
18 \text { a } 35 \text { anos no Hospital } \\
\text { Beneficente Portuguesa de } \\
\text { Belém do Pará/PA, Brasil }\end{array}$ & $\begin{array}{lr}\text { Revista } & \text { Científica } \\
\text { Multidisciplinar } & \text { Núcleo do } \\
\text { Conhecimento }\end{array}$ \\
\hline Neiva et al., 2003 & 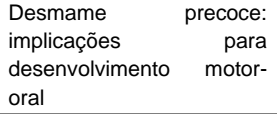 & Jornal de Pediatria \\
\hline Richter et al., 2016 & $\begin{array}{l}\text { Utilização de } \\
\text { medicamentos por } \\
\text { gestantes usuárias do } \\
\text { Sistema Único de Saúde } \\
\text { em um município do Vale } \\
\text { do Taquari-RS }\end{array}$ & $\begin{array}{l}\text { Revista } \\
\text { Acadêmicos }\end{array}$ \\
\hline
\end{tabular}

A partir da análise dos artigos e de acordo com os objetivos do estudo elaborou-se três categorias temáticas:

a. O uso de psicofármacos na gravidez e lactação

b. As complicações dos psicotrópicos

c. Os fármacos mais seguros a serem utilizados por gestantes

\section{a. O uso de psicofármacos na gravidez e lactação}

Costa et al..$^{10}$ e Alberto e Cancellier ${ }^{11}$ concordam que a segurança do uso de psicofármacos durante a gestação ainda não foi estabelecida. Ainda, citam que a decisão de instituir terapêutica psicofarmacológica deve ser tomada sempre com base na gravidade da doença mental e apenas quando o risco potencial para o feto resultante da exposição à terapêutica for superado pelo risco do não tratamento da perturbação materna.

Com o objetivo de guiar os médicos quanto à segurança das drogas prescritas durante a gestação, o FDA (Food and Drug Administration) estabeleceu um sistema que classifica as medicações em cinco categorias de risco, como mostra a tabela 2.

Tabela 2. Categoria de risco de medicamentos durante a gestação adaptada da FDA (Food and Drug Administration).

\begin{tabular}{|c|c|}
\hline CLASSIFICAÇÃO & EVIDËNCIA \\
\hline A & $\begin{array}{l}\text { Medicamentos para os quais não foram } \\
\text { constatados riscos para o feto em } \\
\text { ensaios clínicos cientificamente } \\
\text { desenhados e controlados }\end{array}$ \\
\hline B & $\begin{array}{l}\text { Medicamentos para os quais os estudos } \\
\text { com animais de laboratório não } \\
\text { demonstraram risco fetal (mas não } \\
\text { existem estudos adequados em } \\
\text { humanos) e medicamentos cujos estudos } \\
\text { com animais indicaram algum risco, mas } \\
\text { que não foram comprovados em } \\
\text { humanos em estudos devidamente } \\
\text { controlados }\end{array}$ \\
\hline C & $\begin{array}{l}\text { Medicamentos para os quais os estudos } \\
\text { em animais de laboratório revelaram } \\
\text { efeitos adversos ao feto, mas não } \\
\text { existem estudos adequados em } \\
\text { humanos e medicamentos para os quais } \\
\text { não existem estudos disponíveis }\end{array}$ \\
\hline D & $\begin{array}{l}\text { Medicamentos para os quais a } \\
\text { experiência de uso durante a gravidez } \\
\text { mostrou associação com o aparecimento } \\
\text { de más-formaçoses, mas que a relação } \\
\text { risco-benefício pode ser avaliada }\end{array}$ \\
\hline $\mathbf{X}$ & $\begin{array}{l}\text { Medicamentos associados com } \\
\text { anormalidades fetais em estudos com } \\
\text { animais e em humanos e ou cuja relação } \\
\text { risco-benefício contraindica seu uso na } \\
\text { gravidez }\end{array}$ \\
\hline
\end{tabular}

Com relação à lactação, alguns estudos sugerem que o receio materno de usar medicamentos durante a amamentação e a escassez de informações na literatura sobre a segurança dos fármacos são pretextos utilizados para o desmame precoce ${ }^{12}$. No entanto, para Neiva et al. ${ }^{13}$, esse fato deveria se justificar apenas quando o medicamento em questão fosse contraindicado, já que o aleitamento materno é de fundamental importância tanto para a mãe quanto para a criança.

O desmame, muitas vezes está relacionado ao desconhecimento do grau de segurança dos fármacos e daí vem a importância da avaliação da terapia medicamentosa da mãe e a incessante busca na literatura científica dos riscos potenciais para a saúde materna e infantil. Visando isso, o Ministério da Saúde produziu um manual que agrupa os fármacos de acordo com a categoria de risco na amamentação (Tabela 3). 
Tabela 3. Categoria de risco durante a amamentação segundo o Ministério da Saúde.

\begin{tabular}{cl}
\hline USO COMPATÍVEL COM A & $\begin{array}{l}\text { Uso é potencialmente seguro durante } \\
\text { a lactação, haja vista não haver relatos } \\
\text { de efeitos farmacológicos } \\
\text { significativos para o lactente }\end{array}$ \\
\hline USO CRITERIOSO DURANTEATE A & $\begin{array}{l}\text { Uso no período da lactação depende da } \\
\text { avaliação do risco/benefício }\end{array}$ \\
AMAMENTAÇÃO & $\begin{array}{l}\text { Drogas que exigem a interrupção da } \\
\text { amamentação, pelas evidências ou risco } \\
\text { significativo de efeitos colaterais } \\
\text { importantes no lactente }\end{array}$ \\
\hline USO CONTRAINDICADO DURANTE \\
AMAMENTAÇÃO
\end{tabular}

\section{b. As complicações dos psicotrópicos}

Anton e Mariano ${ }^{14}$, citam que durante a gestação é possível utilizar antidepressivos tricíclicos (ADTs), entre eles amitriptilina, clomipramina, desipramina e nortriptilina, evitando-se preferencialmente no primeiro trimestre. Contudo, não há relatos de associação significativa entre ADTs e malformações congênitas descritos até o momento, mesmo no primeiro trimestre. Os ADTs devem ser suspensos duas semanas antes do parto, a fim de evitar problemas cardíacos, irritabilidade, desconforto respiratório, espasmos musculares, convulsões ou retenção urinária em neonatos.

Ferrão et al. ${ }^{15}$ cita ainda que o uso no terceiro trimestre dos antidepressivos tricíclicos, pode estar associado a risco de síndrome de abstinência no recém-nascido, com sintomas como tremores, irritabilidade e dificuldade com a alimentação e o sono. Alguns estudos sugerem que os ADTs causam algumas complicações gestacionais, como prematuridade, baixo peso ao nascer e pré-eclâmpsia. Estudos longitudinais, entretanto, demonstraram que crianças expostas no período intrauterino a antidepressivos tricíclicos têm inteligência global e desenvolvimento motor, comportamental e de linguagem dentro da normalidade.

$\mathrm{Na}$ amamentação, O ADT maprotilina deve ser evitado por apresentar uma meia vida relativamente longa. Contudo, os outros fármacos ADTs, com exceção da doxepina, não têm sido detectados em doses altas no soro de lactentes de mães que tenham feito uso destes medicamentos, nem têm sido relatados efeitos colaterais significativos ${ }^{14}$.

Com relação aos inibidores seletivos de recaptação de serotonina (ISRSs) - fluoxetina, paroxetina, sertralina, citalopram e escitalopram - Ferrão et al..$^{15}$, afirma que assim como os antidepressivos tricíclicos, os ISRSs usados durante o terceiro trimestre podem levar a complicações neonatais, como agitação, irritabilidade, choro contínuo, alteração do sono e da alimentação, vômitos, diarreia, hipoglicemia, hipotermia, desconforto respiratório e tremores.

Por outro lado, a fluoxetina tem se associado a alguns casos de acatisia, especialmente quando a dose é muito alta. Também, a estimulação do SNC parece maior com a fluoxetina que com outros ISRS. Tem-se relatado sintomas de abstinência através da supressão brusca do tratamento com a paroxetina e com a venlafaxina ${ }^{14}$.
$\mathrm{Na}$ lactação, a maioria dos relatos dos outros ISRS envolvem paroxetina e sertralina. Quanto a primeira, não foi detectável em qualquer soro infantil, não havendo evidência de efeitos adversos de curto prazo. Já a fluoxetina e o citalopram são distribuídos para o leite materno em níveis terapêuticos, e seu uso em lactantes não é recomendado ${ }^{14}$.

Dentre os ansiolíticos, Valadares ${ }^{16}$ afirma que os benzodiazepínicos devem ser evitados no primeiro trimestre da gravidez, em especial da sexta à nona semana, quando ocorre o fechamento dos lábios e da fenda palatina, e se muito necessários devem ser usados nas menores doses possíveis. Em contrapartida, Gusso ${ }^{17}$ afirma que o uso dos benzodiazepínicos deve ser evitado tanto durante a gestação quanto no período de amamentação, por seu efeito sedativo no feto e no lactente.

Costa et al..$^{10}$ alerta que em relação ao alprazolam não existem relatos formais de teratogenicidade (risco C). A exposição ao lorazepam tem sido associada a atresia anal e a sintomas de abstinência neonatal, tais como baixos scores de Apgar, depressão respiratória, hipotermia, dificuldade na sucção e icterícia. Os estudos em relação ao uso do diazepam durante o primeiro trimestre de gestação são controversos e estão associados a risco aumentado de lábio leporino e fenda palatina.

O clonazepam apresenta relativa segurança e recebe a classificação $\mathrm{C}$. O seu uso durante a gestação tem sido associado a doença cardíaca congênita, defeito do septo ventral, deslocação da anca, obstrução da junção úteropélvica, hérnia inguinal bilateral, íleo paralítico do intestino delgado, cianose, letargia e hipotonia ${ }^{10}$.

Para Duncan ${ }^{18}$, uso de doses altas por tempo prolongado pode levar ao aparecimento de sintomas de abstinência no neonato (hipertonia, hiperreflexia, irritabilidade, inquietação, choro inconsolável, tremores de extremidades, bradicardia, cianose, dificuldade de sucção, apneia, diarreia, vômitos). Se usados próximo ao parto, podem levar à síndrome "floppy baby" (hipotonia, hipotermia, letargia, depressão respiratória e dificuldades de alimentação). Os benzodiazepínicos também parecem estar associados a abortos espontâneos.

Dentre os estabilizadores de humor, destaca-se o lítio, o ácido valpróico e a carbamazepina. Tanto Blaya et al. ${ }^{19}$ quanto Costa et al. ${ }^{10}$, relatam que o lítio tem sido associado a malformações, principalmente cardiovasculares, em especial a anomalia de Ebstein (hipoplasia do ventrículo direito e implantação baixa da válvula tricúspide). Afirmaram ainda que evidenciaram casos de toxicidade neonatal como distúrbios do ritmo cardíaco, dificuldade respiratória, cianose, diabetes insipidus nefrogênico, disfunção da tireoide, hipoglicemia, hipotonia, letargia, hiperbilirrubinemia e bebês ditos GIG (grandes para idade gestacional). Brasil ${ }^{20}$ alerta que o lítio tem uso criterioso durante a amamentação. Deve-se monitorizar os níveis séricos no lactente e observar sinais como inquietação, fraqueza e hipotermia no lactente.

Para Blaya et al..$^{19}$ e Costa et al. ${ }^{10}$, o valproato de sódio (VPA) é considerado um provável teratógeno humano quando usado durante o primeiro trimestre de gestação. Ressaltaram também sobre a chamada "Síndrome do 
Valproato Fetal", caracterizada por anomalias crâniofaciais com hipoplasia da região média da face, micrognatia, implantação baixa das orelhas, fenda palatina e hipertelorismo. Costa et al. ${ }^{10}$ afirma ainda que quando usado próximo ao parto tem sido associado a complicações neonatais como desacelerações da taxa cardíaca, toxicidade hepática, hipoglicemia, reduções nos níveis de fibrinogênio neonatais e sintomas de abstinência como irritabilidade, icterícia, dificuldades alimentares e tônus anormal. Blaya et al..$^{19} \mathrm{e} \mathrm{Brasii^{20 }}$ asseguram que o VPA tem uso compatível com a amamentação.

A carbamazepina tem sido associada a um risco de 2 a 3 vezes superior de malformações cardíacas, especialmente de espinha bífida, quando utilizada nos dois primeiros trimestres da gestação. Pode ainda associar-se a fenda platina, atresia anal, meningomielocele, genitália ambígua e a chamada face anticonvulsivante, caracterizada por hipoplasia da região média da face, nariz curto e lábio superior longo ${ }^{10}$. Brasil ${ }^{20}$ ratificam que a carbamazepina tem uso compatível com a amamentação.

Dentre os antipsicóticos, destacam-se o haloperidol e as fenotiazidas (levomepromazina, clorpromazina). Costa et al. ${ }^{10}$ assegura que a maioria dos estudos não encontrou aumento de malformações fetais em crianças expostas ao haloperidol (antipsicótico de alta potência) durante o primeiro trimestre de gestação. $O$ seu uso em doses elevadas e por períodos extensos pode associar-se a sintomas extrapiramidais no recém-nascido, podendo este vir a apresentar hiperatividade, movimentos anormais e tremores. Os antipsicóticos de baixa potência como a clorpromazina deverão ser evitados, decorrente dos seus efeitos colaterais, tais como hipotensão.

\section{c. Os fármacos mais seguros a serem utilizados por gestantes}

Para Blaya et al. ${ }^{19}$, a nortriptilina, devido ao menor efeito anticolinérgico, é o ADT de escolha na gestação. Ferrão et al. ${ }^{15}$ ressaltam que os inibidores seletivos da recaptação de serotonina são considerados relativamente seguros durante a gestação. Esses são os fármacos mais comumente utilizados ao redor do mundo, o que faz com que os dados sobre seu uso sejam mais robustos em comparação com as demais classes de antidepressivos.

Sertralina e paroxetina são os antidepressivos dessa classe de preferência durante a amamentação, apresentando-os como fármacos mais seguros do que a fluoxetina e o citalopram. O nível da medicação no leite materno é baixo e não é detectado no soro do lactente ${ }^{14}$.

Blaya et al. ${ }^{19}$, alertou que se não for possível evitar o uso de benzodiazepínicos durante a gestação, deve-se optar por aqueles de meia-vida mais curta como o alprazolam e o lorazepam. Uma vez que os BDZ são detectados no leite, essa classe de drogas deve ser evitada durante a lactação. Brasil ${ }^{20}$ afirma que se deve evitar o uso benzodiazepínico em mulheres grávidas e no período pós-natal.

Entre os antipsicóticos disponíveis, a evidência de segurança é superior para os de elevada potência. De entre os antipsicóticos de alta potência, o haloperidol é o mais bem estudado em relação ao seu uso durante a gestação. Apesar da possibilidade de induzir efeitos extrapiramidais no recém-nascido, os antipsicóticos de alta potência são preferíveis na gestação, sendo mínimos os efeitos adversos maternos anticolinérgicos, antihistamínicos e hipotensores ${ }^{10}$.

A tabela 4 apresenta os psicofármacos e suas classificações, segundo a FDA para o período da gestação e o Ministério da Saúde para período de aleitamento.

Tabela 4. Classificação dos psicofármacos.

\begin{tabular}{|c|c|c|}
\hline PSICOFÁRMACOS & $\begin{array}{l}\text { CLASSIFICAÇÃO } \\
\text { FDA (GESTAÇÃO) }\end{array}$ & $\begin{array}{l}\text { CLASSIFICAÇÃO } \\
\text { MINISTÉRIO DA } \\
\text { SAÚDE } \\
\text { (ALEITAMENTO) }\end{array}$ \\
\hline \multicolumn{3}{|l|}{ ADTs } \\
\hline Amitriptilina & B & Uso compatível com \\
\hline Nortriptilina & B & a amamentação \\
\hline Clomipramina & C & \\
\hline Desipramina & C & \\
\hline Doxepina & C & Uso contraindicado \\
\hline \multirow[t]{2}{*}{ Maprotilina } & B & $\begin{array}{ll}\text { durante } & \text { a } \\
\text { amamentação }\end{array}$ \\
\hline & & $\begin{array}{l}\text { Uso criterioso } \\
\text { durante } \\
\text { amamentação }\end{array}$ \\
\hline \\
\hline $\begin{array}{l}\text { Sertralina } \\
\text { Citalopram }\end{array}$ & B & Uso compatível com \\
\hline \multirow[t]{2}{*}{ Paroxetina } & $\begin{array}{l}B \\
C\end{array}$ & a amamentação \\
\hline & D & \\
\hline \multicolumn{3}{|l|}{ BENZODIAZEPINICOS } \\
\hline Diazepam & D & $\begin{array}{l}\text { Uso compatível com } \\
\text { a amamentação em } \\
\text { doses esporádicas. }\end{array}$ \\
\hline Clonazepam & C & \\
\hline Alprazolam & C & criterioso \\
\hline Lorazepam & C & $\begin{array}{ll}\text { durante } & \text { a } \\
\text { amamentação }\end{array}$ \\
\hline
\end{tabular}

ESTABILIZADORES DO

HUMOR

$\begin{array}{lllr}\text { Carbonato de lítio } & \text { D } & \text { Uso } & \text { criterioso } \\ \text { Carbamazepina } & \text { C } & \text { amamentação } \\ \text { Valproato de sódio } & \text { D } & \end{array}$

\section{CONCLUSÃO}

$\mathrm{Na}$ literatura, a segurança do uso dos psicofármacos durante a gestação e lactação ainda não foi estabelecida. Entretanto, alguns estudos apontaram alguns fármacos menos teratogênicos, tanto para o bebê como para a mãe.

Dentre os ADTs e os ISRS, tem-se como fármacos mais seguros tanto na gestação como lactação, a nortriptilina e a sertralina, respectivamente. Com relação a classe dos ansiolíticos, os benzodiazepínicos devem ser evitados tanto na gravidez como na amamentação. Porém, se não tiver nenhuma outra opção, deve-se optar por aqueles de meia-vida mais curta. Além deles, os 
estabilizadores de humor carbonato de lítio, carbamazepina e valproato de sódio também devem ser evitados. Dentre os antipsicóticos, observou-se que o haloperidol é o mais estudado, sendo o preferido na gestação e lactação.

Desse modo, concluiu-se que a nortriptilina (ADTs), sertralina (ISRS) e o haloperidol (antipsicótico), são os fármacos mais seguros a serem usados durante o período de gravidez e lactação.

A utilização desses medicamentos nesse período é um processo de decisão complexo, por isso, é fundamental o estabelecimento de um vínculo terapêutico, envolvendo uma interação constante entre doente, família, obstetra e psiquiatra. A equiparação de riscos e benefícios durante qualquer intervenção na gestação, deve levar em consideração a mãe e o feto, buscando sempre a melhor escolha para esse binômio.

\section{REFERÊNCIAS}

1. BRASIL, Ministério da Saúde. Secretaria de Atenção à Saúde. Departamento de Ações Programáticas Estratégicas Pré-natal e puerpério: atenção qualificada e humanizada. Brasília - DF, 2005

2. BRASIL, Ministério da Saúde. Secretaria de Atenção à Saúde. Departamento de Atenção Básica. Atenção ao pré-natal de baixo risco / Secretaria de Atenção à Saúde. Departamento de Atenção Básica. - Brasília, 2013

3. ROSANA, Márcia; SARTURI, Fernanda. Uma revisão sobre os problemas emocionais e as orientações e intervenções em saúde mental na assistência pré-natal. Revista Saúde. Santa Maria, v. 38, n. 1, p. 31-46, 2012

4. MOREIRA, Elisangela et al. Incidência de transtornos psíquicos na gravidez e puerpério em mulheres de 18 a 35 anos no Hospital Beneficente Portuguesa de Belém do Pará / PA, Brasil. Revista Científica Multidisciplinar Núcleo do Conhecimento. São Paulo, $v$. 2, p. $81-93,2016$

5. NOGUEIRA, Luiz, et al. Assistência psiquiátrica no ciclo gravídicopuerperal. Psicologia na prática obstétrica: abordagem interdisciplinar. Barueri, Manole, p. 118-122, 2007.
6. PEREIRA, Priscila; LOVISI, Giovanni. Prevalência da depressão gestacional e fatores associados. Rev. Psiq. Clín. Rio de Janeiro, 35(4):144-53, 2008

7. FERREIRA, Suely. Uso de psicofármacos durante a gravidez e lactação: uma revisão bibliográfica. 30f. (Monografia apresentada ao Curso de Especialização em Linhas de Cuidado em Enfermagem em Atenção Psicossocial). Florianópolis, UFSC, 2014.

8. FERNANDES, Naiana. Assistência Farmacêutica em gestantes. $93 f$ (Trabalho de Conclusão de Curso de graduação em FarmáciaBioquímica). Araraquara, UNESP, 2013

9. SCIORILLI, Renata, et al. Transtornos psiquiátricos na gestação e no puerpério: classificação, diagnóstico e tratamento. Rev. Psiq. Clín. São Paulo, 33 (2); 92-102, 2006.

10. COSTA, Cassilda; REIS, Constança; COELHO, Rui. Uso de psicofármacos na gravidez. Acta Obstet Ginecol Port, Porto, 4(2): p. 101-111, 2010

11. ALBERTO, Carlos; CANCELLIER, Kelen. Antidepressivos na ginecologia e obstetrícia. Revista Psychiatry online Brasil. São Paulo, v. 22, n. 3, 2017

12. MARLON, Jefferson; GUEDES, Andreza. Uso de medicamentos durante a lactação: um fator para suspensão do aleitamento materno. Revista de Pesquisa Interdisciplinar. Cajazeiras, no 2, p. 504-513, setembro. 2017.

13. NEIVA, Flávia et al. Desmame precoce: implicacões para o desenvolvimento motor-oral. Jornal de Pediatria. Jundiaí, v. 79, n. 1 2003.

14. ANTON, Raquel; MARIANO, Rafael. Avaliação da segurança no uso de antidepressivos na amamentação. Revista Interdisciplinar de Estudos em Saúde. Caçador, v. 6, n. 2, p. 103-117, 2017.

15. FERRÃO, Ygor et al. Psicofármacos na gravidez e na lactação. In CORDIOLLI, A. V.; GALLOIS, C. B.; ISOLAN, L. (Org.) Psicofármacos: consulta rápida. 5 ed. Porto Alegre: Artmed, 2015.

16. VALADARES, Gislene. Uso de psicofármacos na gravidez e no pósparto. Revista Brasileira de Psiquiatria. Rio de Janeiro, ed. 6, 2006. Disponível em: http://www.abpbrasil.org.br/boletim/exibBoletim/?bol id=6\&boltex id=2 4>. Acesso em: 15/04/2018.

17. GUSSO, Gustavo; LOPES, José. Tratado de Medicina de Família e Comunidade: princípios, formação e prática. 2 ed. Porto Alegre: ArtMed, 2019.

18. DUNCAN, Bruce et al. Medicina Ambulatorial: Condutas de Atenção Primária Baseadas em Evidências. 4 ed. Porto Alegre: ArtMed, 2014.

19. BLAYA, Carolina et al. Diretrizes para o uso de psicofármacos durante a gestação e lactação. Porto Alegre, 2005.

20. BRASIL, Ministério da Saúde. Secretaria de Atenção à Saúde. Departamento de Ações Programáticas e Estratégias. Amamentação e uso de medicamentos e outras substâncias. Brasília - DF, 2014. 